\title{
Tres nuevas especies del grupo guarani, género Drosophila (Diptera, Drosophilidae) en el Bosque Pasochoa, Provincia de Pichincha
}

\author{
Doris Vela y Violeta Rafael
}

Laboratorio de Genética Evolutiva.

Departamento de Ciencias Biológicas, Pontificia Universidad Católica del Ecuador, 17-01-2184, Quito-Ecuador.

RESUMEN. En el Bosque Pasochoa, se encontraron tres nuevas especies del género Drosophila las cuales son descritas en el presente artículo.

ABSTRACT. In Pasochoa Forest, tree new species of Drosophila genus were found, they are described in the present paper.

PALABRAS CLAVE. grupo guara$n i$, nuevas especies, Drosophila, Pasochoa, Ecuador.

\section{INTRODUCCIÓN}

$\mathrm{E}$ (1) con el objetivo de agrupar a cuatro especies de Sudamérica, a las que posteriormente se agregó una especie de Centroamérica y otra del sur de México (2). El análisis de la morfología externa, la terminalia, el aislamiento sexual y los cromosomas politénicos de estas seis especies del grupo guarani (D. griseolineata Duda, 1925; D. orna- tifrons Duda, 1925; D. guaru Dobzhansky \& Pavan, 1943; D. guaramunu Dobzhansky \& Pavan, 1943; D. subbadia Patterson, 1943 y D. guaraja King, 1947) planteó la existencia de dos subgrupos dentro del grupo guarani: el subgrupo guarani formado por D. guarani, D. guaru y D. subbadia, y el subgrupo guaramunu formado por D. guaramunu, $D$. griseolineata y $D$. guaraja. Estudios de los cromosomas politénicos de cuatro de estas especies también mostraron la existencia de diferencias en la morfología cromosómica de cada una de ellas, sugiriendo que en el grupo guarani estarían incluidos dos grupos naturales de especies (3).

Además de las diferencias a nivel citológico y etológico, dentro del grupo guarani existe tres especies (D. peruensis VILELA Y PEREIRA, 1985; D. tucumana VIlela Y PeREIRA, 1985 y D. urubamba VILELA Y PEREIRA, 1993) que se distinguen del resto por la presencia de manchas en el tórax (VILELA, comunicación oral).

Hasta 1993 se conocía la existencia de 13 especies dentro del grupo guara$n i$, sin embargo hay que señalar que de 
D. peruensis se conoce únicamente la terminalia de la hembra $(4,5)$.

\section{MATERIALES Y MÉTODOS}

Moscas del género Drosophila colectadas durante 1996 y 1997 en una hectárea del Bosque Pasochoa, fueron, capturadas usando trampas de plátano fermentado y trasladadas al laboratorio en tubos con medio de cultivo gelatinabanano (6). Se analizó la morfología y la terminalia de machos y hembras, también se calcularon los índices del edeago y de la espermateca, estas mismas estructuras fueron dibujadas y fotografiadas en un microscopio con cámara fotográfica, en aumento 20x. Los datos que aparecen entre paréntesis en las descripciones de las nuevas especies, corresponden a los rangos observados en los paratipos analizados.

Holotipo y paratipos de cada especie se conservan en el Museo de Zoología, Pontificia Universidad Católica del Ecuador [QCAZ] en Quito.

\section{RESULTADOS}

\section{Drosophila ecuatoriana sp. nov.}

(Figs. 1-9,20).

Drosophila ecuatoriana (nomen nudum); RAFAeL \& VELA 2000: 88.

Material tipo. Holotipo o 7 (disectado, terminalia en microtubo), etiquetado D. ecuatoriana holotipo ?

D. Vela det. 1999 / Pasochoa, Pichincha-Ecuador D. Vela col., Jul. 1996. Nueve paratipos $\sigma^{7}$ (disectados, terminalia en microtubos), etiquetados $D$. ecuatoriana paratipo D. Vela det. 1999 / Pasochoa, Pichincha-Ecuador D. Vela col., Jul. 1996. Tres paratipos o (disectados, terminalia en microtubos), etiquetados D. ecuatoriana paratipo o D. Vela det. 1999 / Pasochoa, Pichincha-Ecuador D. Vela col., Ago. 1996. Dos paratipos o (disectados, terminalia en microtubos), etiquetados D. ecuatoriana paratipo of D. Vela det. 1999 / Pasochoa, Pichincha-Ecuador D. Vela col., Jul. 1997. Siete paratipos of (disectados, terminalia en microtubos), etiquetados $D$. ecuatoriana paratipo of D. Vela det. 1999 / Pasochoa, Pichincha-Ecuador D. Vela col., Jul. 1996. Un paratipo o (disectados, terminalia en microtubos), y un paratipo $\sigma$, etiquetados D. ecuatoriana paratipo o y D. ecuatoriana paratipo $\sigma^{7}$ respectivamente D. Vela det. 1999 / Pasochoa, Pichincha-Ecuador D. Vela col,, Jul. $1997 /$ colectados en cópula en la naturaleza.

Localidad tipo. ECUADOR, Pichincha: Volcán Pasochoa, 35 km. al sur de Quito.

Descripción. Cuerpo de color marrón, longitud del cuerpo $3.4 \mathrm{~mm}$, cabeza de color marrón claro, dos cerdas orales, ojos de color rojo oscuro, arista con 5-7 ramas superiores y 3 ramas inferiores más la bifurcación terminal. Alas sombreadas en dm-Cu, longitud $3.8 \mathrm{~mm}\left(\sigma^{\pi}\right), 4.2 \mathrm{~mm}\left(\sigma^{\pi}\right)$. índice costal $3.66\left(\sigma^{\pi}\right), 4.2\left(\sigma^{\sharp}\right)$. Tórax de color marrón claro polinoso, con 6 filas irregulares de pelos acrostiçales entre las 

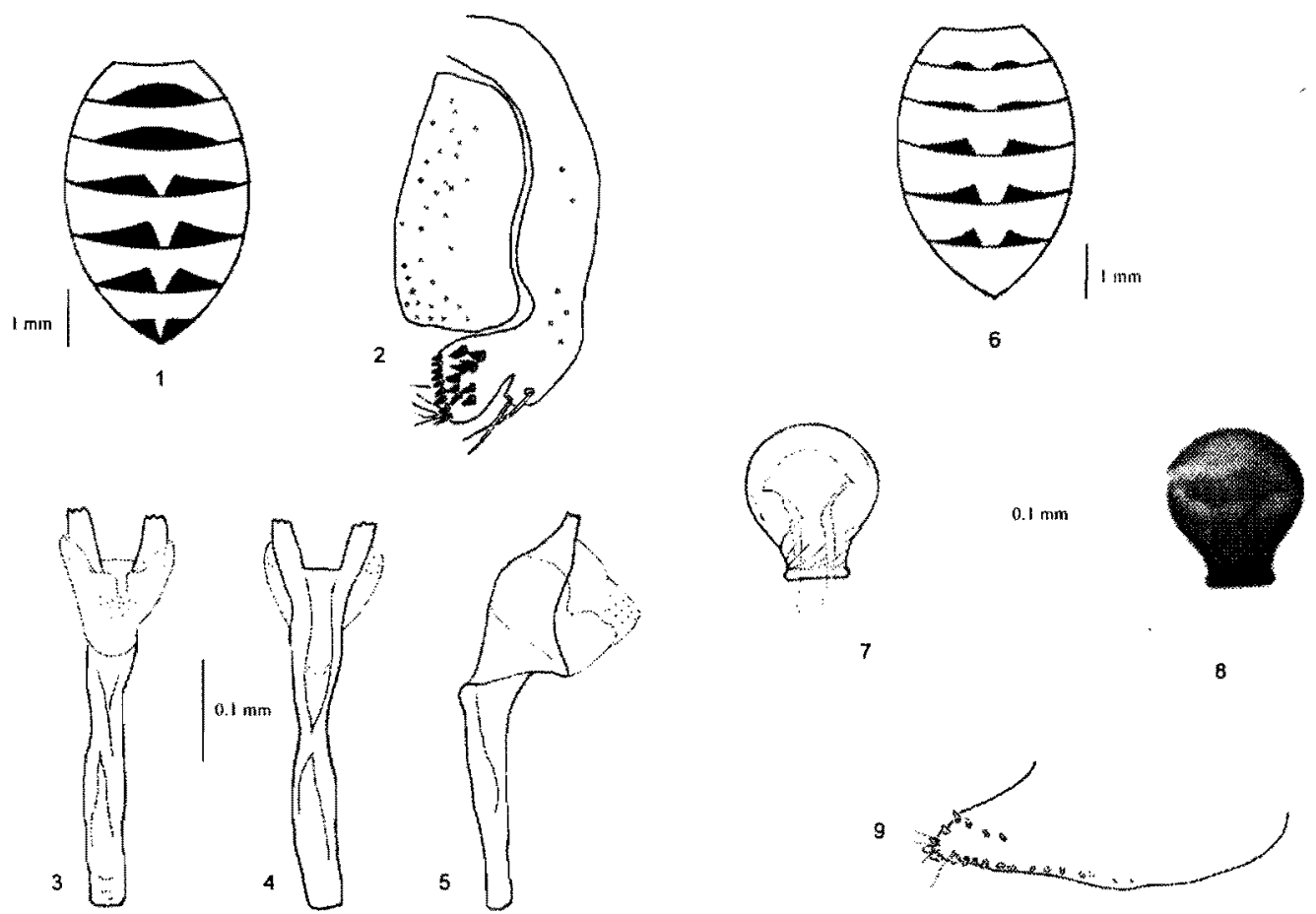

7

8

Figs. 1-5. Drosophila ecuatoriana sp. nov., holotipo macho: 1, patrón de pigmentación abdominal; 2, epandrio, placa anal y surestilo, vista posterior, lado izquierdo omitido; 3-5, edeago y apodema del edeago, vista ventral, dorsal y lateral izquierda respectivamente, gonopodio omitido.

Figs. 6-9. Drosophila ecuatoriana sp. nov., paratipo hembra: 6, patrón de pigmentación abdominal; 7,8 , espermateca en vista lateral; 9 , ovipositor, vista lateral derecha.

cerdas dorsocentrales, sin cerdas preescutelares.

Cerdas escutelares anteriores divergentes. Abdomen marrón, en los machos la línea dorsal media se presenta del tercer al sexto tergito, en el primero y segundo tergitos la pigmentación forma un semicírculo, del tercer tergito al sexto las bandas marginales se extienden lateralmente (fig. 1), en las hembras la línea dorsal media está en todo los tergitos y las bandas marginales tie- nen forma de triángulos (fig. 6).

Terminalia o : Placa anal libre. Surestilo con 9 (8-12) dientes primarios, $8(6-9)$ dientes secundarios y $9(7-12)$ cerdas marginales; $2(2-6)$ cerdas en la parte baja del epandrio (fig. 2).

Edeago quitinizado, en la parte apical presenta dos proyecciones con el borde aserrado, en la parte ventral presenta una membrana replegada con microproyecciones en la zona externa, índice del edeago 0.79 (figs. 3-5, 20). 
Algunos paratipos presentan micropubescencia en la parte quitinizada del edeago, gonopodio poco quitinizado, unido al edeago por tejido membranoso.

Terminalia $q$ : Espermateca muy quitinizada de forma redonda con un borde en la base y la superficie enrolla$\mathrm{da}$, conducto medianamente invaginado (figs. 7,8).

Ovipositor quitinizado con 5 dientes discales, 18 cerdas marginales y 4 espinas, una larga y tres cortas, índice de la espermateca 0.42. (fig. 9).

Etimología. Esta especie ha sido nombrada para hacer honor a la República del Ecuador, donde han sido descubiertas varias nuevas especies del género Drosophila

\section{Drosophila pichinchana sp. nov.}

(Figs. 10-14, 21).

Drosophila pichinchana (nomen nudum); RAFAel \& Vela 2000: 88.

Material tipo. Holotipo ${ }^{7}$ (disectado, terminalia en microtubo), etiquetado D. pichinchana holotipo o 7 D. Vela det. 1999 / Pasochoa, Pichincha-Ecuador D. Vela col., Jul. 1996. Un paratipo $\sigma^{7}$ (disectado, terminalia en microtubo), etiquetado $D$. pichinchana paratipo of D. Vela det. 1999 / Pasochoa, Pichincha-Ecuador D. Vela col., Jul. 1996.

Localidad tipo. ECUADOR, Pichincha: Volcán Pasochoa, $35 \mathrm{~km}$. al sur de Quito.

Descripción. Longitud del cuerpo $3.0 \mathrm{~mm}$. Cuerpo de color marrón, ca- beza de color marrón claro, una cerda oral de tamaño mediano, carina sin surco. Arista con cuatro ramas superiores, una rama inferior más la bifurcación terminal. Alas sombreadas en dm$\mathrm{Cu}$, longitud $3.9 \mathrm{~mm}$. Índice costal 4.5. Tórax de color marrón claro. Seis filas de pelos acrosticales entre las cerdas dorsocentrales, sin cerdas preescutelares.

Cerdas escutelares anteriores divergentes. Abdomen amarillo, línea media dorsal presente del primer tergito al quinto y en cada uno hay bandas marginales oscuras de forma triangular que no llegan a los bordes laterales, el sexto tergito está pigmentado en la parte central (fig. 10).

Terminalia of: Placa anal libre y micropubescente, especialmente en la zona inferior. Surestilo con 10-12 (10-11) dientes primarios 11-12 (9-12) dientes secundarios y 11 (9-12) cerdas marginales; 5-6 (5-9) cerdas quitinizadas en la parte baja del epandrio (fig. 11).

Edeago quitinizado, en la parte apical presenta cuatro proyecciones quitinizadas, dos dorsales gruesas y dos ventrales delgadas, una región membranosa en la parte media la misma que presenta microproyecciones (visibles en $40 \mathrm{x}$ ), gonopodio poco quitinizado, unido al edeago por tejido membranoso, índice del edeago 0.9. (figs. 12-14, 21).

Etimología. Esta especie es nombrada en alusión a la provincia de Pichincha en la cual se encuentra el volcán Pasochoa. 


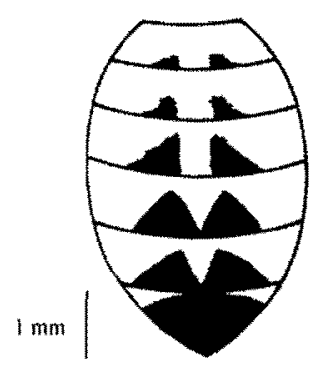

10

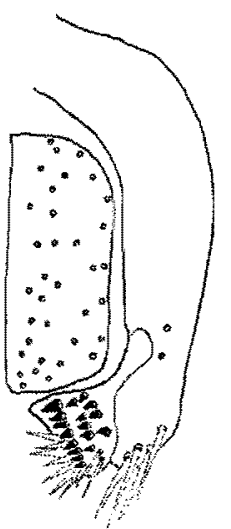

11
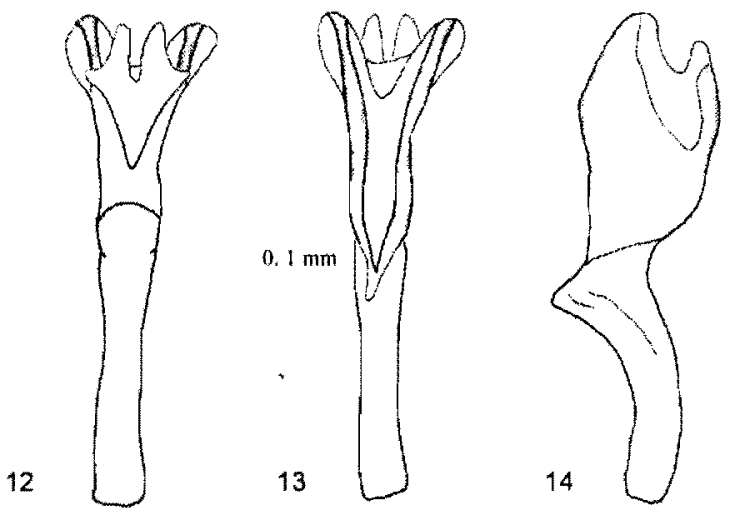

Figs. 10-14. Drosophila pichinchana sp. nov., holotipo macho: 10, patrón de pigmentación abdominal; 11, epandrio, placa anal y surestilo, vista posterior, lado izquierdo omitido; $12-14$, edeago y apodema del edeago, vista ventral, dorsal y lateral izquierda respectivamente, gonopodio omitido.

Drosophila quitensis sp. nov.

(Figs. 15-19, 22).

Drosophila quitensis (nomen nudum); RAFAEL \& VELA 2000: 88.

Material tipo. Holotipo o ${ }^{\prime}$ (disectado, terminalia en microtubo), etiquetado D. quitensis holotipo o ${ }^{7} \mathrm{D}$. Vela det. 1999 / Pasochoa, Pichincha-Ecuador D. Vela col., Jul. 1996. Dos paratipos $\delta$ (disectados, terminalia en microtubos), etiquetados $D$, quitensis paratipo
Of D. Vela det. 1999 / Pasochoa, Pichincha-Ecuador D. Vela col., Ago. 1996.

Localidad tipo. ECUADOR, Pichincha: volcán Pasochoa, $35 \mathrm{~km}$. al sur de Quito.

Descripción. Longitud del cuerpo $3.0 \mathrm{~mm}$. Cuerpo de color marrón oscuro, cabeza de color marrón oscuro, ojos marrón. Arista con cinco ramas superio- 


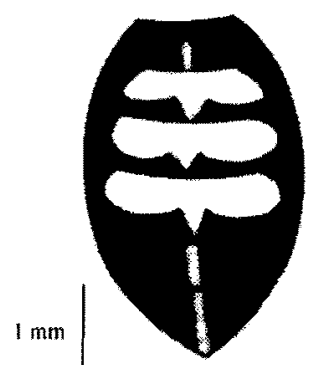

15
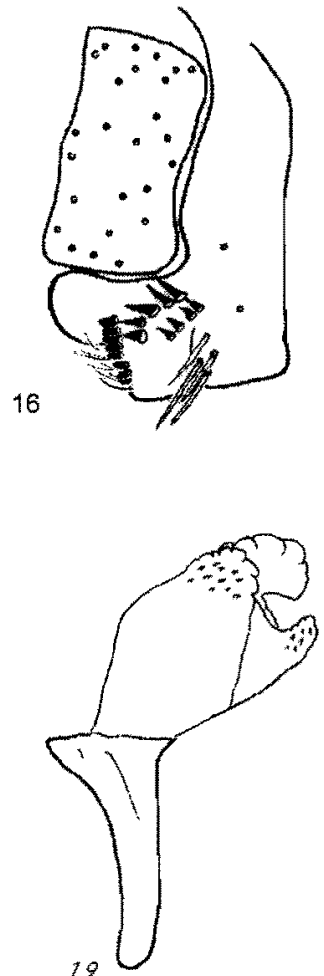

Figs. 15-19. Drosophila quitensis sp. nov., holotipo macho: 15, patrón de pigmentación abdominal; 16, epandrio, placa anal y surestilo, vista posterior, lado izquierdo omitido; $17-19$, edeago y apodema del edeago, vista ventral, dorsal y lateral izquierda respectivamente, gonopodio omitido.

res y una rama inferior más la bifurcación terminal. Alas sombreadas en $\mathrm{r}-\mathrm{m} \mathrm{y}$ $\mathrm{dm}-\mathrm{Cu}$, longitud $3.5 \mathrm{~mm}$. indice costal 4.06. Tórax de color marrón oscuro.

Cerdas escutelares anteriores convergentes. Abdomen marrón oscuro, la línea dorsal media presente en todos los tergitos, del segundo tergito al cuarto la parte central superior no está pigmentada, el primero, quinto y sexto tergitos son totalmente pigmentados excepto en la línea media dorsal (fig. 15).

Terminalia of : Placa anal libre y mi- cropubescente. Hipandrio más pequeño que epandrio. Surestilo con 8 (6-8) dientes primarios; $9(6-10)$ dientes secundarios y 8 (6-11) cerdas marginales; 4 cerdas en la parte baja del epandrio (fig. 16).

Edeago quitinizado, en la parte api$\mathrm{cal}$ se presentan cuatro proyecciones, las dos proyecciones dorsales son muy quitinizadas, presentan los bordes aserrados $\mathrm{y}$ excrecencias, las dos proyecciones ventrales son pequeñas, membranosas y presentan microproyecciones, gonopo- 
dio poco quitinizado, unido al edeago por tejido membranoso, índice del edeago 1.18 (figs. 17-19, 22).

Etimología. Esta especie lleva el nombre de la ciudad de Quito, capital de la República del Ecuador.

\section{DISCUSIÓN}

Los estudios realizados en el Bosque Protector Pasochoa, un bosque andino del Ecuador, han permitido el descubrimiento de tres nuevas especies del grupo guarani: Drosophila ecuatoriana sp. nov., Drosophila pichinchana sp. nov. y Drosophila quitensis sp. nov. Anteriormente se conocía la presencia de tres especies de este grupo: $D$. urubamba (citada como $D$. peruensis) (7) y $D$. griseolineata en Monteserrin Bajo, otro bosque andino de la Provincia de Pichincha, y D. ornatifrons (citada como D. guarani) (6).
En los primeros estudios filogenéticos del género Drosophila sitúan al grupo guarani dentro de la sección quinaria-tripunctata (8), por otro lado, en base al análisis de cuatro especies del grupo guarani, se propone la división de este grupo en dos grupos naturales, cuyas especies no presentan similitudes cromosómicas y por el contrario, dos de ellas, $D$. griseolineata y D. guaramu$n u$, serían más cercanas a algunas especies del grupo tripunctata que a las del grupo guarani $(2,3)$.

También algunos cambios de agrupación se han producido en el grupo guarani, por ejemplo: $D$. urubamba fue agrupada en primera instancia en el grupo repleta debido a la presencia de manchas en el tórax y luego transferida al grupo guarani (9), por otro lado $D$. neoguaramunu Frydenberg, 1959 fue trasladada del grupo guarani al grupo tripunctata (10).

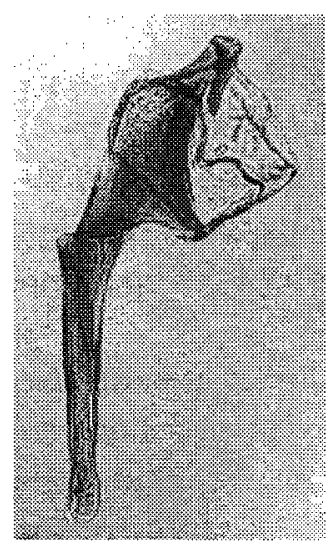

20

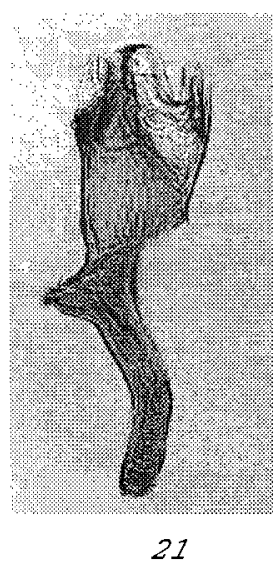

21

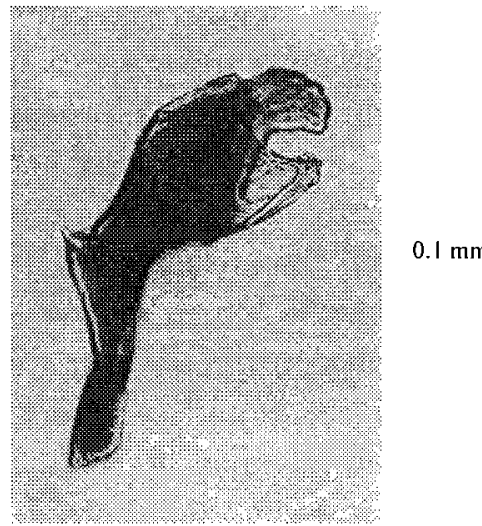

22

Figs. 20-22. Edeago y apodema del edeago en vista lateral de: 20, Drosophila ecuatoriana sp. nov. holotipo macho; 21, Drosophila pichinchana sp. nov. holotipo macho; 22, Drosophila quitensis sp. nov. holotipo macho. 
Los fundamentos para la separación de las especies del grupo guarani en dos grupos naturales y las relaciones entre estas especies deben ser estudiadas con mayor profundidad.

Se desconoce aún la biología de la mayoría de especies, incluidas las nuevas especies descritas en el presente trabajo.

Estudios biológicos de las especies del grupo guarani deberían ser promovidos con el fin de entender sus relaciones evolutivas, lo cual permitiría aceptar o descartar la propuesta de Kastritsis sobre la división de este grupo.

\section{Agradecimientos.}

A la Dra. Laura Arcos Terán, Decana de la Facultad de Ciencias Exactas y Naturales y a la Lcda. Ana Beatriz Mafla profesora de la PUCE. A IDEA Wild por la donación del material entomológico.

\section{REFERENCIAS}

1. Dobzhansky, T. \& PAVAN, C. Studies on Brazilian species of Drosophila. Bol. Facult. Filos. Cien. Letras Univ. Sao Paulo, 1943; 36: 7-72.

2. KING, ]. C. Interspecific relationships within the guarani group of Drosophila. Evolution, 1947; 1:143-153.

3. Kastritsis, C. D. The chromosomes of some species of the guarani group of Drosopbila. J. Heredity, 1969; 60:51-57.

4. Vilela, C. R. \& Pereira, M. A. Notes on two species of spot-tho- raxed Drosophila belonging to the guarani group (Diptera, Drosophilidae). Revista Brasileira de Entomología, 1985; 29 (3-4):435-442.

5. Vilela, C. R. \& Pereira, M. A. A case of misidentification of a neotropical species of Drosophila (Diptera, Drosophilidae) belonging to the guarani group. Revista Brasileira de Entolomogía, 1993; 37(4):819-820.

6. RAFAel, V; G. ARcos \& L. ARCOS. Ecología y distribución del Género Drosopbila en Guayllabamba y el Quinche, provincia de Pichincha-Ecuador. Revista de la Pontificia Universidad Católica del Ecuador, Quito, 2000; 65:130-155.

7. Rafael, V. \& Vela, D. Drosophila distribution in Ecuador. Drosophila information Service, Norman, 2000; 83:85-88.

8. Trockmorton, L. Philogeny in Drosophila. University Texas $\mathrm{Pu}-$ blications. Austin, 1962; 6205: 207-343.

9. VILELA, C. R. A revisión of the Drosophila repleta species group (Diptera, Drosophilidae). Revista Brasileira de Entomologia, 1983; 27(1):1-114.

10. VILELA, C.R. \& BÄCHLI, G. Taxonomic studies on Neotropical species of seven genera of Drosophilidae (Diptera). Bulletin de la Société Entomologique Suisse, 1990; 64:1-332. 